\title{
Schlingensysteme dauerhaft wirksam bei Inkontinenz
}

- Wie eine Inkontinenz behandelt wird, hängt von der genauen Art des Leidens ab. In leichten Fällen können bei einer Belastungsinkontinenz schon Verhaltensänderungen wie Beckenbodentraining helfen. Oft ist auch eine chirurgische Behandlung mit Implantation einer Schlinge bei männlicher und auch bei weiblicher Inkontinenz eine dauerhafte Lösung.

Mit ihrem einzigartigen Vier-Arm-System bietet die Virtue ${ }^{\oplus}$-Schlinge gegenüber anderen Schlingen für Männer deutliche Vorteile. Sie vereint erstmals zwei Therapieprinzipien nach einer Prostata-Operation: Das Vier-Arm-Design und das starke Netz aus $100 \%$ MonofilPolypropylen ermöglichen eine bidirektionale
Kompression und gleichzeitig eine Anhebung der bulbären Harnröhre. Durch die sanfte Ausübung von Druck wird dem Urinaustritt vorgebeugt. Dank der innovativen Implantationstechnik muss keine chirurgische Dissektion bis zur Harnröhre vorgenommen werden. So wird das Risiko chirurgischer Komplikationen minimiert und der Patient kann schneller als bei anderen Verfahren seine Blase wieder normal entleeren.

Eine minimalinvasive Kontinenzlösung für Frauen besteht im adjustierbaren SingleIncision-Schlingen-System Altis ${ }^{\oplus}$. Es erlaubt die Platzierung eines suburethralen Bandes mit nur einem Einschnitt. Die Altis ${ }^{\circledR}$-Schlinge besteht aus monofilen Fäden aus Polypropy- len. Zwei stärkere Fäden führen zu den beiden zur Schlinge gehörenden Gewebeankern: einem statischen Anker und einem zur Anpassung bidirektional verschiebbaren. Damit lässt sich die Schlinge während der Operation individuell an die anatomischen Gegebenheiten der Patientin anpassen und sorgt so dauerhaft für eine optimale Unterstützung der Urethra. Die Gewebeanker wurden individuell für maximale Haltekraft und minimale Gewebeverletzung entwickelt. Die zum Schlingensystem gehörenden Einführhilfen ermöglichen eine Kontrolle bei der Positionierung der Gewebeanker.

Nach Informationen von Coloplast, Hamburg

\section{GnRH-Blocker: IQUO-Studie bestätigt klinische Daten}

- Die Ergebnisse einer nicht interventionellen Studie (NIS) zu Wirkung und Sicherheit des GnRH-Blockers Degarelix (Firmagon ${ }^{\circledR}$ ) ergänzen und bestätigen die bereits vorliegenden klinischen Daten bei Patienten mit Prostatakarzinom. In die NIS der Arbeitsgruppe um Dr. Götz Geiges, Berlin, vom IQUO (Interessenverband zur Qualitätssicherung der Arbeit niedergelassener Uro-Onkologen in Deutschland) wurden die Daten von $421 \mathrm{~Pa}$ tienten aufgenommen [Geiges $\mathrm{G}$ et al. Alkaline phosphatase control and prostate volume reduction under degarelix treatment in prostate cancer patients confirmed by real life data from a German registry. Poster AUA 2012]. Die Analysen waren vorgeplant und wurden retrospektiv durchgeführt. Hinsichtlich des PSAWertes betrug der mediane Ausgangswert 12,8 ng/ml. Die größte Gruppe machten bei der Verteilung des PSA-Wertes Patienten mit einem Ausgangs-PSA-Wert $<10 \mathrm{ng} / \mathrm{ml}$ aus. In dieser Gruppe wurde nach 12 Monaten bei $83 \%$ der Patienten eine Senkung dieses Parameters auf $<4 \mathrm{ng} / \mathrm{ml}$ erzielt. Auch bei Patienten mit einem Ausgangs-PSA-Wert $>50 \mathrm{ng} /$ ml wurde der PSA-Wert schnell und anhaltend unterdrückt. Nach dreimonatiger Therapie verringerte sich das Prostatavolumen durchschnittlich um 40,8\%. Dieses Ergebnis stützt die Daten einer früheren Vergleichsstudie von Degarelix gegenüber Leuprorelin.

In der IQUO-Studie hatte Degarelix Knochenmetastasen gut unter Kontrolle: Die alkalische
Phosphatase als Marker für Knochenmetastasen wurde schnell gesenkt und blieb bis zum Messzeitpunkt "Monat 12" auf niedrigem Niveau. Das Ergebnis bestätigt die Daten einer früheren Phase-III-b-Studie [Schröder FH et al. BJU Int 2010; 106: 182-7].

Von Degarelix profitierten ebenfalls Patienten, die bereits vor der Behandlung mit dem GnRH-Blocker eine Hormontherapie erhalten hatten. Auch in dieser Subgruppe wurden die Testosteronwerte schnell und anhaltend gesenkt.

Degarelix erhöhte auch die Lebensqualität. So berichteten die Patienten bei der letzten Untersuchung über eine um 20,9\% reduzierte Schmerzfrequenz und über eine um 58,8\% verringerte Häufigkeit an Rückenschmerzen.

Nach Informationen von Ferring, Kiel

\section{Mit der Heilkraft der Natur gegen Blasenentzündungen}

— Wichtigstes Therapieziel bei Harnwegsinfektionen ist die Bekämpfung der Bakterien als Infektursache. Eine schonende und gleichzeitig effektive Möglichkeit bietet hier der Trockenextrakt aus Bärentraubenblättern (Cystinol akut ${ }^{\oplus}$ Dragées).„Bei akuten, unkomplizierten Harnwegsinfekten ist, wenn diese auf die Blase begrenzt sind, die Einnahme eines Antibiotikums nicht zwingend erforderlich", sagte Dr. Michael Zellner, Bad Füssing. Er erläuterte:„Antibiotika haben negative Auswirkungen auf die physiologische Keimflora von Darmtrakt und Scheide und können so die lokale oder systemische Immunabwehr beeinträchtigen. Durch eine antibiotische Behandlung eines akuten Harnwegsinfekts erhöht sich häufig das Risiko, in Folge einen weiteren Infekt zu erleiden." Der Trockenextrakt aus Bärentraubenblättern wirkt antibakteriell, antientzündlich und verhindert ein Anheften der Bakterien an die Blasenschleimhaut. Die Bakterien werden zuverlässig bekämpft, die Beschwerden gebessert und die entzündete Schleimhaut kann sich schnell erholen. Eine Antibiotikatherapie kann so meist vermieden werden.

Nach Informationen von Schaper \& Brümmer, Salzgitter 